\title{
DECOMPOSING 3-SPACE INTO CIRCLES AND POINTS
}

\author{
RONALD H. ROSEN ${ }^{1}$
}

1. Introduction. R. H. Bing and M. L. Curtis have shown that if $G$ is an upper semicontinuous decomposition of $E^{n}$ whose only nondegenerate elements are two compact continua, then the decomposition space $G$ associated with $G$ can be imbedded in $E^{n+1}$, [1]. Accordingly a decomposition of $E^{3}$ into two disjoint circles and points not on the circles can be imbedded in $E^{4}$. In the same paper Bing and Curtis exhibited a decomposition of $E^{3}$ into twelve mutually disjoint circles and points not on the circles so that the decomposition space could not be imbedded in $E^{4}$. The method was to show that the space contained a 2-dimensional polyhedron, the Menger polyhedron $M_{2}$, which Flores in [2] had shown could not be imbedded in $E^{4}$. It was conjectured in [1] that if the only nondegenerate elements were three circles linked by pairs, then the decomposition space could not be homeomorphically mapped into $E^{4}$. R. P. Goblirsch proved, however, that this conjecture was false (see [3]). Below, by modifying the construction of Bing and Curtis and slightly strengthening Flores' result, we give an example of a decomposition of $E^{3}$ into six circles and the points not on the circles so that the decomposition space cannot be imbedded in $E^{4}$.

This paper formed part of a dissertation [4] written at the University of Wisconsin under the direction of R. H. Bing. The author wishes to express his gratitude to Professor Bing for his encouragement and for directing his attention to these problems. This research was supported in part by the National Science Foundation.

2. Description of the Bing-Curtis example. Consider the geometric 2-complex $M_{2}$, the Menger 2-polyhedron, consisting of three triples of vertices $a_{i}, b_{i}, c_{i}, i=1,2,3$, and all triangles of the form $\left(a_{i}, b_{j}, c_{k}\right)$. In Figure 1 is depicted a construction by which Bing and Curtis imbed in $E^{3}$ the subpolyhedron of $M_{2}$ consisting of all triangles of $M_{2}$ except those of the form $\left(a_{i}, b_{i}, c_{i}\right), i=1,2,3$.

We will describe the construction in stages. Take a circular disk in the $(x, y)$ plane and mark off in order at equal distances on its perimeter the points $a_{1}, b_{2}, a_{3}, b_{1}, a_{2}$, and $b_{3}$. Denote by $c_{2}$ the center

Presented to the Society, April 17, 1959 under the title A decomposition of 3-space that cannot be imbedded in 4-space; received by the editors January 4, 1960.

1 The author thanks the referee and the Editor for their suggestions and has incorporated some of these in the paper. 
of the disk. Taking radii from $c_{2}$ to each of the six designated points divides the disk into six sectors $\left(a_{i}, b_{j}, c_{2}\right), i \neq j$, which correspond to triangles in $M_{2}$.

Choose two points on the perpendicular to the $(x, y)$ plane through $c_{2}$ which lie at a distance from $c_{2}$ equal to the radius of the disk, $c_{1}$ above $c_{2}$ and $c_{3}$ below $c_{2}$. Now take the spherical double cone over the

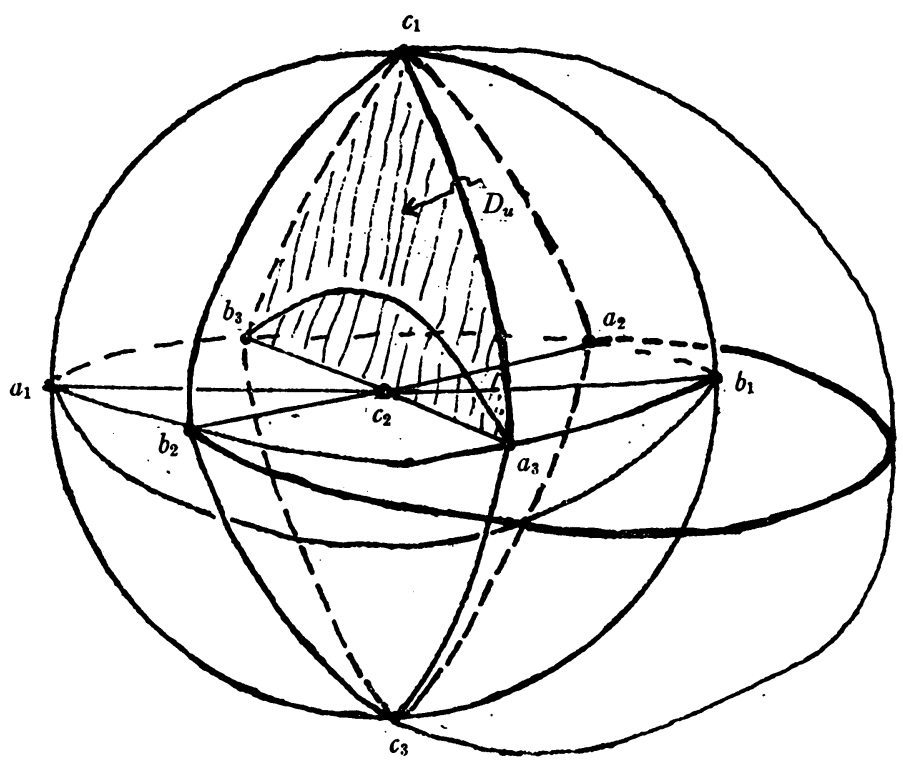

FIGURE 1

boundary of the disk with vertices $c_{1}$ and $c_{3}$. The upper hemisphere is divided into six spherical triangles $\left(a_{i}, b_{j}, c_{1}\right), i \neq j$; the lower hemisphere, into "triangles" $\left(a_{i}, b_{j}, c_{3}\right) i \neq j$.

The curvilinear complex so far constructed in $E^{3}$ has three complementary domains, an unbounded domain $W$ and two bounded domains, $U$ above the $(x, y)$ plane, $V$, below.

Let $D_{U}$ be the open disk which is the intersection of $U$ with the plane through the 1-simplices $\left(b_{3} c_{2}\right),\left(c_{2} a_{3}\right),\left(a_{3} c_{1}\right)$, and $\left(c_{1} b_{3}\right)$. In $D_{U} \cup a_{3} \cup b_{3}$ take a circular arc with endpoints $a_{3}$ and $b_{3}$. Similarly utilizing a planar open disk $D_{V}$ in $V$, we fit the "triangles" $\left(a_{3}, b_{3}, c_{2}\right)$, $\left(a_{3}, b_{3}, c_{1}\right),\left(a_{1}, b_{1}, c_{2}\right)$, and $\left(a_{1}, b_{1}, c_{3}\right)$ into the polyhedron.

In the $(x, y)$ plane take a circular arc in $W$ with endpoints $a_{2}$ and $b_{2}$. By taking curvilinear cones over $\left(a_{2} b_{2}\right)$ from $c_{1}$ and $c_{3}$, we fit the "triangles" $\left(a_{2}, b_{2}, c_{1}\right)$ and $\left(a_{2}, b_{2}, c_{3}\right)$ into the complex. 
We now cut out the interiors of nine disks and add three annular rings to this complex, as follows: In $\left(a_{3}, b_{3}, c_{1}\right)$ cut out the interior of a disk $D_{1}$ so that the boundary curve $J_{1}$ intersects the boundary of $\left(a_{3}, b_{3}, c_{1}\right)$ precisely in $c_{1}$. In the triangles $\left(a_{1}, b_{2}, c_{2}\right)$ and $\left(a_{3}, b_{1}, c_{2}\right)$ cut out disks $D_{2}$ and $D_{3}$ whose boundaries $J_{2}$ and $J_{3}$ intersect the boundaries of their respective triangles in $a_{1}$ and $b_{1}$. Using the holes cut out of the figure, an annular ring $A_{1}$ is placed with one boundary curve along the 1-chain $\left(a_{1} b_{1}\right)\left(b_{1} c_{1}\right)\left(c_{1} a_{1}\right)$ so that $A_{1}$ intersects the figure only along one of its boundary curves. Let the free boundary curve be called $K_{1}$. See Figure 2 .

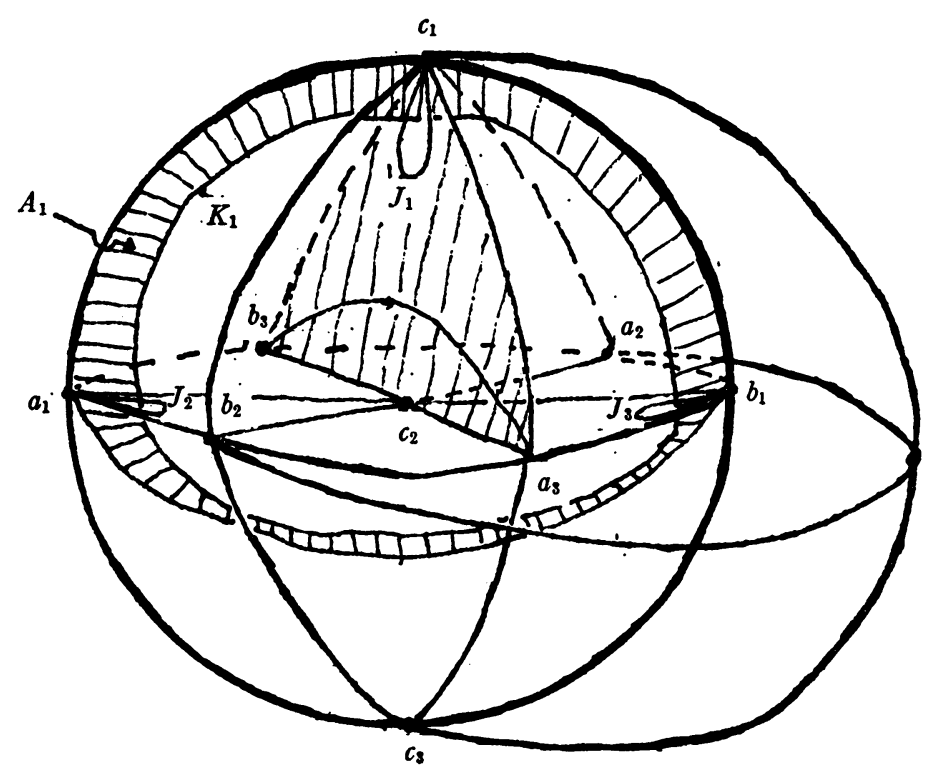

FIGURE 2

In a similar manner by cutting out disks $E_{1}, E_{2}, E_{3}$ with boundaries $J_{1}^{\prime}, J_{2}^{\prime}, J_{3}^{\prime}$ intersecting the 1-skeleton of the figure only in $a_{2}, b_{2}, c_{2}$ respectively, we add an annular ring $A_{2}$ with one boundary curve along $\left(a_{2} b_{2}\right)\left(b_{2} c_{2}\right)\left(c_{2} a_{2}\right)$ and with a free boundary curve $K_{2}$. An annulus $A_{3}$ is fitted along the edges $\left(a_{3} b_{3}\right)\left(b_{3} c_{3}\right)\left(c_{3} a_{3}\right)$ running through cut-out disks $F_{1}, F_{2}, F_{3}$, with boundaries $J_{1}^{\prime \prime}, J_{2}^{\prime \prime}, J_{3}^{\prime \prime} . A_{3}$ intersects the previous figure only along the aforementioned edge path, and we call its free boundary curve $K_{3}$. We call $P$ the polyhedron so obtained.

We note that $K_{1}, K_{2}, K_{3}$ are linked by pairs, $J_{i}$ links $K_{1}$ but none of the other added curves, $J_{i}^{\prime}$ links $K_{2}$, and $J_{i}^{\prime \prime}$ links $K_{3}$. The twelve simple closed curves $K_{i}, J_{i}, J_{i}^{\prime}, J_{i}^{\prime \prime}(i=1,2,3)$ are all tame, and there 
is a homeomorphism of $E^{3}$ onto itself taking the twelve curves onto a collection of twelve circles linked, of course, in a similar manner. This implies that the decomposition space described by using these twelve curves as nondegenerate elements is homeomorphic to one in which the nondegenerate elements are circles.

The Bing-Curtis example is the decomposition of $E^{3}$ into the twelve curves and the remaining points. The decomposition space contains a homeomorph of $M_{2}$, namely the image of $P$. Identifying the curves $J_{i}, J_{i}^{\prime}, J_{i}^{\prime \prime}$ to points closes up the holes made by cutting out $D_{i}, E_{i}$, $F_{i}$; identifying the curves $K_{i}$ to points adds in the triangles $\left(a_{i}, b_{i}, c_{i}\right)$, $i=1,2,3$, in the decomposition space.

We can improve the example to one with only nine nondegenerate elements whose decomposition space still contains $M_{2}$. We eliminate $J_{1}, J_{1}^{\prime}, J_{1}^{\prime \prime}$ by the device of using "pinched annuli" for the $A_{i}$, that is sets homeomorphic to a disk with two points on the boundary identified; the idea is that such a figure still has two boundary curves such that if either is identified to a point, the identification image is again a disk. For example throwing away $J_{1}$, we leave $\left(a_{3}, b_{3}, c_{1}\right)$ untouched and pass the pinched annulus $A_{1}$ with one boundary curve on $\left(a_{1} b_{1}\right)\left(b_{1} c_{1}\right)\left(c_{1} a_{1}\right)$ through the holes at $D_{2}$ and $D_{3}$. The other simple closed curve $K_{1}$ on the boundary of $A_{1}$ is free except at the point $c_{1}$. Identifying $K_{1}, J_{2}, J_{3}$ to points gives back all the triangles as before. The decomposition space with the nine curves $K_{1}, K_{2}, K_{3}, J_{i}, J_{i}^{\prime}$, $J_{i}^{\prime \prime}(i=2,3)$ as nondegenerate elements is the example mentioned.

3. Our example using six curves. It seems impossible to construct a decomposition space containing $M_{2}$ using fewer than nine circles. To construct the example promised in the Introduction, we modify the original Bing-Curtis twelve curve example as follows. We cut out only the disks $D_{1}, E_{1}$ and $F_{3}$ bounded by the curves $J_{1}, J_{1}^{\prime}$ and $J_{3}^{\prime \prime}$, eliminating the curves $J_{i}, J_{i}^{\prime}, J_{i-1}^{\prime \prime}, i=2,3$. We relabel $J_{1}, J_{1}^{\prime}$ and $J_{3}^{\prime \prime}$ simply as $J, J^{\prime}$ and $J^{\prime \prime}$. The annuli $A_{i}$ described above with edges $K_{i}$ are retained. We call $Q$ the polyhedron so obtained. We consider now the decomposition of $E^{3}$ in which the six curves $J, J^{\prime}, J^{\prime \prime}$, $K_{i}, i=1,2,3$, are the nondegenerate elements. The image of $Q$ in the decomposition space we denote by $N$.

$N$ is a polytope consisting of the union of twenty-seven topological triangles labelled in the same way as the triangles of $M_{2}$. We shall describe a map $f$ of $M_{2}$ onto $N$ which has the following property: for each pair of disjoint closed 2-simplexes $\sigma$ and $\tau, f(\sigma)$ and $f(\tau)$ are also disjoint. $f$ maps the boundary of a 2 -simplex $\left(a_{i} b_{j} c_{k}\right)$ isomorphically onto the boundary of the corresponding triangle in $N$ with vertices going into corresponding vertices. Note that $f$ restricted to the 
1-skeleton of $M_{2}$ is an isomorphism. The annulus $A_{1}$ intersects the triangle $\left(a_{1}, b_{2}, c_{2}\right)$ in $Q$ in a line segment $r_{1}$ which joins a point on $K_{1}$ (the free boundary of $A_{1}$ ) to the vertex $a_{1}$. Except for one endpoint $r_{1}$ lies in the interior of $\left(a_{1}, b_{2}, c_{2}\right)$. Similarly $A_{1}$ intersects $\left(a_{3}, b_{1}, c_{2}\right)$ in a segment $r_{2}$ which joins a point on $K_{1}$ to the vertex $b_{1}$; except for one endpoint, $r_{2}$ lies in the interior of $\left(a_{3}, b_{1}, c_{2}\right)$. We extend $f$ to map homeomorphically the interior of the simplex $\left(a_{1}, b_{1}, c_{1}\right)$ in $M_{2}$ onto the interior of the image of $A_{1}$ in $N$ in such a fashion that the barycenter $\beta$ goes into the image of $K_{1}$ and the closed line segments $s_{1}=a_{1} \beta$ and $s_{2}=b_{1} \beta$ go onto the images of $r_{1}$ and $r_{2}$ in $N$, respectively. In a similar fashion $f$ takes the interior of $\left(a_{1}, b_{2}, c_{2}\right)$ in $M_{2}$ onto the image in $N$ of the interior of the triangle $\left(a_{1}, b_{2}, c_{2}\right)$ from $Q$; the segment in $M_{2}$ which connects the vertex $a_{1}$ with the barycenter of $\left(a_{1}, b_{2}, c_{2}\right)$ is mapped onto the image of $r_{1}$ in $N$. Similarly $f$ is extended to the interior of $\left(a_{3}, b_{1}, c_{2}\right)$ in $M_{2}$, mapping the segment which joins the barycenter of this simplex to the vertex $b_{1}$ onto the image of $r_{2}$ in $N$.

Let us now describe the self-intersections caused by $f$ on the images of the simplexes mentioned. $f\left(a_{1}, b_{1}, c_{1}\right)$ meets $f\left(a_{1}, b_{2}, c_{2}\right)$ exactly in the image of $r_{1} ; f\left(a_{1}, b_{1}, c_{1}\right)$ meets $f\left(a_{3}, b_{1}, c_{2}\right)$ precisely in the image of $r_{2} ; f\left(a_{1}, b_{2}, c_{2}\right)$ meets $f\left(a_{3}, b_{1}, c_{2}\right)$ only in the two points $f\left(c_{2}\right)$ and the point in $N$, corresponding to the curve $K_{1}$, which is also the image of the barycenters of the three 2-simplexes. Notice that the selfintersections are produced only in the images of the interiors of the simplexes and that in $M_{2}$ each pair of these simplexes has a vertex in common.

$f$ can be extended analogously over the remaining simplexes of $M_{2}$ in such a way that the desired property is preserved. The theorem proved in the next section guarantees that $N$ cannot be imbedded in $E^{4}$ since if we let the $C, f$, and $D$ used in the statement of the theorem be $M_{2}, f$ and $N$, the hypotheses of the Theorem are satisfied for $n=2$. Since our example contains $N$, it in turn cannot be imbedded in $E^{4}$.

We note that each pair of $K_{1}, K_{2}, K_{3}$ are linked and $J, J^{\prime}, J^{\prime \prime}$ link only $K_{1}, K_{2}, K_{3}$, respectively. The decomposition of $E^{3}$ using these six curves is equivalent to a decomposition of $E^{3}$ using six circles similarly linked. We will complete the proof that this six curve decomposition space cannot be imbedded in $E^{4}$ by proving in the next section that $N$, the singular image of $M_{2}$, cannot be imbedded in $E^{4}$.

4. A strengthening of Flores' result. In order to obtain a proof of the theorem required, we have found it necessary to reprove many of the results mentioned in Flores' paper [2], which is essentially an 
outline. For the sake of completeness we include our own proofs; the form of the argument, i.e. the statements of many of the lemmas, is due to Flores. Our final theorem strengthens Flores' main result and is proved in an analogous manner.

Consider $A \times B \times I$, the Cartesian product of two disjoint spaces $A$ and $B$ with the unit interval $[0,1]$. The join of $A$ and $B$, which is designated by $V(A, B)$, is an identification space derived from $A \times B \times I$ as follows. For each $a \in A$, identify $(a, B, 0)$ with $a$. For each $b \in B$, identify $(A, b, 1)$ with $b$. Thus $A$ and $B$ are imbedded in $V(A, B)$, which may be thought of as a continuous collection of arcs connecting each pair of points one of which is from $A$, the other from $B$. Let $\phi$ be the identification function sending $A \times B \times I$ onto $V(A, B)$. The latter is topologized to make $\phi$ continuous, i.e. $U$ is open in $V(A, B)$ if $\phi^{-1}(U)$ is open.

We consider the join operation to be symmetric so that $V(A, B)$ and $V(B, A)$ are the same space. If $B$ is a point $p$, then $V(A, p)$ is also called the (topological) cone over $A$ with vertex $p$. If $B$ is a 0 sphere, $V(A, B)$ is called the suspension of $A$ or the double cone over $A$ with vertices in $B$. It is easy to see that $V(A, p)$ is homeomorphic to $A \times I$ with $(A, 1)$ identified as a point. Similarly $S(A)$, the suspension of $A$, is equivalent to $A \times[-1,1]$ with $(A,-1)$ and $(A, 1)$ identified to separate points. It is also quickly verified that $V(A, B)$ $-(A \cup B)$ is homeomorphic to $A \times B \times(0,1)$.

If two spaces $X$ and $Y$ are homeomorphic, we shall write $X \approx Y$. For $n \geqq 0, S^{n}$ will always mean the boundary of the unit ball in $E^{n+1}$.

Lemma 1. $V\left(S^{p}, S^{q}\right) \approx S^{p+q+1}$.

Proof. This is, of course, well known, but later we shall need to use the particular homeomorphism described below.

Let us consider $S^{p}$ and $S^{q}$ imbedded in $E^{p+q+2}$ in the following way,

$$
\begin{aligned}
& S^{p}=\left\{x \mid x=\left(x_{1}, \cdots, x_{p+1}, 0, \cdots, 0\right),\|x\|=1\right\}, \\
& S^{q}=\left\{y \mid y=\left(0, \cdots, 0, y_{1}, \cdots, y_{q+1}\right),\|y\|=1\right\} .
\end{aligned}
$$

If $(x, y, t) \in V\left(S^{p}, S^{q}\right)$, let $\phi(x, y, t)=(1-t)^{1 / 2} x+t^{1 / 2} y . \phi$ is a homeomorphism of $V\left(S^{p}, S^{q}\right)$ onto $S^{p+q+1}$.

By a complex $C$ we will always mean a countable locally finite complex; $|C|$ will be the underlying space with the barycentric metric. We will suppose that there is a fixed ordering $v_{1}, v_{2}, v_{3}, \ldots$ on the set of vertices of $C$ which will be designated as $C_{v}$. Simplices will be considered to be closed. It will be understood that if a subcomplex of $C$ contains a simplex $\sigma$, it contains the faces of $\sigma$.

Suppose $K$ and $L$ are disjoint complexes. The join of $K$ and $L$, 
$K \circ L$, is a complex consisting of all simplices $\sigma$ such that $\sigma_{v} \subseteq K_{v} \cup L_{v}$ and $\sigma_{v} \cap K_{v}, \sigma_{v} \cap L_{v}$ are vertices of a simplex in $K, L$, respectively.

The next lemma is, again, well known, but the particular homeomorphism described will be used later.

Lemma 2. Let $K$ and $L$ be disjoint complexes. Then $V(|K|,|L|)$ $\approx|K \circ L|$.

Proof. Given any pair of simplices $\sigma \in K, \tau \in L$, we will define a homeomorphism $f: V(\sigma, \tau) \rightarrow|\sigma \circ \tau|$. By the local finiteness of $K$ and $L$ it may be checked that these mappings may be fitted together to give the required homeomorphism.

For each point $x \in V(\sigma, \tau)$, since points of $\sigma, \tau$ have barycentric coordinates in $|K|,|L|$, respectively, $x$ is of the form $\left(\sum \lambda_{i} v_{i}^{K}, \sum \mu_{j} v_{j}^{L}, t\right)$ and $f(x)=(1-t) \sum \lambda_{i} v_{i}^{K}+t \sum \mu_{j} v_{j}^{L} \in|\sigma \circ \tau|$.

Lemma 2 allows us to identify $V(|K|,|L|)$ and $|K \circ L|$ by the homeomorphism $f$, which in turn enables us to use equivalent methods of designating the points of the join of $|K|$ and $|L|$; as we shall see shortly, it is convenient to retain both notations.

Let $C$ be an $n$-dimensional complex and $C^{\prime}$ a disjoint copy of $C$ under an isomorphism $\theta$. Then $U(C)$ will be the subcomplex of $C \circ C^{\prime}$ consisting of all $(2 n+1)$-dimensional simplices $\sigma \circ \tau^{\prime}$ where $\sigma$ and $\tau\left(=\theta^{-1}\left(\tau^{\prime}\right)\right)$ are disjoint $n$-simplices in $C$. We also denote $|U(C)|$ by $U(C)$.

Associated with $C$ is another space $L(C)$ which is a subset of $V(|C|, p) \times V(|C|, p) . L(C)$ consists of all pairs $(\alpha, \beta)$ for which one coordinate lies in a simplex $\sigma$, the other coordinate lies in some $V(\tau, p)$, and $\sigma$ and $\tau$ are disjoint $n$-simplices in $C$. If $(\alpha, \beta) \in L(C)$, then $(\beta, \alpha) \in L(C)$, and the two points are said to be symmetric in $L(C)$.

\section{Lemma 3. $L(C)$ and $U(C)$ are homeomorphic.}

Proof. Flores defines a geometric mapping $g$ of $L(C)$ onto $U(C)$ which is clearly one-to-one and onto. Using the simplicial definition of join we give an equivalent characterization of $g$ in terms of barycentric formulae which exhibit the bicontinuity of $g$ more clearly and which shall also be needed in the next step.

Let $(\alpha, \beta)$ be a point of $L(C)$ for which $\alpha$ and $\beta$ both belong to $|C|$. Then $g$ maps the line segment $(\alpha, V(\beta, p))$ affinely onto half the line $V\left(\alpha, \beta^{\prime}\right)$ connecting $\alpha$ and $\beta^{\prime}(=\theta(\beta))$ in $U(C)$ so that $g((\alpha, p))=\alpha$ and $g((\alpha, \beta))$ is the midpoint $\left(\alpha, \beta^{\prime}, 1 / 2\right)$. Similarly $g$ maps $(V(\alpha, p), \beta)$ affinely onto the other half of the line $V\left(\alpha, \beta^{\prime}\right)$ so that $g((p, \beta))=\beta^{\prime}$.

We will now exhibit formulae for $g$. Suppose $\alpha=\sum \lambda_{i} v_{i} \in \sigma$ and 
$\beta=\sum \mu_{i} v_{i} \in \tau$ where $\sigma$ and $\tau$ are disjoint $n$-simplices of $C$. Then $x=(\alpha,(\beta, p, t))=\left(\sum \lambda_{i} v_{i},(1-t) \sum \mu_{i} v_{i}+t p\right)$ and $g(x)=\left(\alpha, \beta^{\prime},(1-t) / 2\right)$ $=((1+t) / 2) \sum \lambda_{i} v_{i}+((1-t) / 2) \sum \mu_{i} \theta\left(v_{i}\right)$. Also $y=((\alpha, p, t), \beta)$ $=\left((1-t) \sum \lambda_{i} v_{i}+t p, \quad \sum \mu_{i} v_{i}\right)$ and $g(y)=\left(\alpha, \quad \beta^{\prime}, \quad(1+t) / 2\right)$ $=((1-t) / 2) \sum \lambda_{i} v_{i}+((1+t) / 2) \sum \mu_{i} \theta\left(v_{i}\right)$.

If $x, y \in U(C)$ and $g^{-1}(x), g^{-1}(y)$ are symmetric in $L(C)$, we shall call $x$ and $y$ symmetric in $U(C)$. Using the formulae for $g$ we shall find a direct meaning intrinsic to $U(C)$ for two points of $U(C)$ to be symmetric.

Suppose $x=\sum \lambda_{i} v_{i}+\sum \mu_{i} \theta\left(v_{i}\right)\left(\sum \lambda_{i}+\sum \mu_{i}=1\right)$ is a point of $U(C)$; let $\theta(x)=\sum \mu_{i} v_{i}+\sum \lambda_{i} \theta\left(v_{i}\right) . \theta$ is an involution (a period two homeomorphism) of $U(C)$ onto $U(C)$ which we shall call the antipodal map of $U(C) ; x$ and $\theta(x)$ will be called antipodal points of $U(C)$. It is easy to see that if $(1-t) x+t y=z \in U(C), \theta(z)=(1-t) \theta(x)+t \theta(y)$.

LeMma 4. $x$ and $y$ are symmetric in $U(C)$ if, and only if, $x$ and $y$ are antipodal points.

Proof. Let $(\alpha, \beta) \in L(C), \alpha=\sum \lambda_{i} v_{i}, \beta=\sum \mu_{i} v_{i}$. Consider the points $z=(\alpha,(\beta, p, t)), z^{\prime}=((\beta, p, t), \alpha)$ of $L(C)$. Then $g(z)=((1+t) / 2) \sum \lambda_{i} v_{i}$ $+((1-t) / 2) \sum \mu_{i} \theta\left(v_{i}\right), \quad g\left(z^{\prime}\right)=((1-t) / 2) \sum \mu_{i} v_{i}+((1+t) / 2) \sum \lambda_{i} \theta\left(v_{i}\right)$, and $\theta(g(z))=g\left(z^{\prime}\right)$.

Now let $x$ and $y$ be antipodal in $U(C)$. If $x=\sum \lambda_{i} v_{i}, \theta(x)=y$ $=\sum \lambda_{i} \theta\left(v_{i}\right)$, then $g^{-1}(x)=(x, p)$, and $g^{-1}(y)=(p, x)$.

Suppose $x=\sum \lambda_{i} v_{i}+\sum \mu_{i} \theta\left(v_{i}\right), \quad \sum \lambda_{i}+\sum \mu_{i}=1, \quad 1 / 2 \leqq \sum \lambda_{i}<1$. Then $y=\theta(x)=\sum \mu_{i} v_{i}+\sum \lambda_{i} \theta\left(v_{i}\right)$. Equivalently, since $\sum \mu_{i} \leqq 1 / 2$, $x=\left(\left(1 / \sum \lambda_{i}\right) \sum \lambda_{i} v_{i}, \quad\left(1 / \sum \mu_{i}\right) \sum \mu_{i} \theta\left(v_{i}\right), \quad \sum \mu_{i}\right), y=\left(\left(1 / \sum \mu_{i}\right) \sum \mu_{i} v_{i}\right.$, $\left.\left(1 / \sum \lambda_{i}\right) \sum \lambda_{i} \theta\left(v_{i}\right), \sum \lambda_{i}\right)$; accordingly, $g^{-1}(x)=\left(\alpha,\left(\beta, p, 1-2 \sum \mu_{i}\right)\right)$ where $\alpha=\left(1 / \sum \lambda_{i}\right) \sum \lambda_{i} v_{i}, \beta=\left(1 / \sum \mu_{i}\right) \sum \mu_{i} v_{i}$. Similarly since $1 / 2$ $\leqq \sum \lambda_{i}, g^{-1}(y)=\left(\left(\beta, p, 2 \sum \lambda_{i}-1\right), \alpha\right)$, and $1-2 \sum \mu_{i}=2 \sum \lambda_{i}-1$. Hence $g^{-1}(x)$ and $g^{-1}(y)$ are symmetric in $L(C)$. The case in which $1 / 2 \leqq \sum \mu_{i}$ follows in the same way.

A space $X$ will be called absolutely knotted (absolut selbstverschlungen, [2]) with respect to $E^{m}$ if there is no mapping $\phi$ of $V(X, p)$ into $E^{m}$ satisfying these conditions, (a) $\phi$ is one-to-one on $X$ and (b) $\phi(X)$ $\cap \phi(V(X, p)-X)=0$.

Clearly no subset of $E^{m-1}$ is absolutely knotted in $E^{m}$ since $V\left(E^{m-1}, p\right)$ may be imbedded in $E^{m}$. Thus if $X$ is absolutely knotted with respect to $E^{m}, V(X, p)$ cannot be imbedded in $E^{m}$ and $X$ cannot be imbedded in $E^{m-1}$.

Define $M_{0}$ to be a set of three points, and $M_{n}=V\left(M_{n-1}, M_{0}\right), n>0$. $M_{n}$ may be thought of as $n+1$ triples of points $a_{i}, b_{i}, c_{i}, i=1, \cdots$, $n+1$ and all $n$-dimensional simplices having exactly one vertex in each triple. 
Lemma 5. There is a homeomorphism of $S^{2 n+1}$ onto $L\left(M_{n}\right)$ under which antipodal points in $S^{2 n+1}$ correspond precisely to symmetric points in $L\left(M_{n}\right)$.

Proof. We shall actually exhibit a homeomorphism of $S^{2 n+1}$ onto $U\left(M_{n}\right)$ so that antipodal points in $S^{2 n+1}$ correspond to antipodal points in $U\left(M_{n}\right)$. The proof proceeds by induction on $n$.

Let $a, b, c$ be the vertices of an equilateral triangle inscribed in the unit circle $S^{1} \subseteq E^{2}$. Let $\theta(a), \theta(b), \theta(c)=a^{\prime}, b^{\prime}, c^{\prime}$ be diametrically opposite to the corresponding points $a, b, c$. Then, as may be quickly established, $U\left(M_{0}\right)$ is homeomorphic to the hexagon inscribed in $S^{1}$ with vertices $a, b^{\prime}, c, a^{\prime}, b, c^{\prime}$. The desired homeomorphism $h_{0}$ maps $S^{1}$ onto $U\left(M_{0}\right)$ by projection through the center of $S^{1}$.

Now suppose a homeomorphism $h_{n-1}$ of $S^{2 n-1}$ onto $U\left(M_{n-1}\right)$ has been found with the required properties. Flores gives the formula,

$$
\begin{aligned}
U\left(M_{n}\right) & \equiv U\left(V\left(M_{n-1}, M_{0}\right)\right) \equiv V\left(U\left(M_{n-1}\right), U\left(M_{0}\right)\right) \\
& \equiv V\left(S^{2 n-1}, S^{1}\right) \equiv S^{2 n+1} .
\end{aligned}
$$

$U\left(M_{n}\right)$ is the same as $U\left(V\left(M_{n-1}, M_{0}\right)\right)$ by definition.

We may regard $M_{n}$ as the geometric complex consisting of all $n$ dimensional simplices $\sigma \circ a$ so that $\sigma$ is any $(n-1)$-dimensional simplex in $M_{n-1}$ and $a \in M_{0}$. Then $U\left(M_{n}\right)$ may be regarded as the complex consisting of all $(2 n+1)$-dimensional simplices $(\sigma \circ a)$ $\circ(\theta(\tau) \circ \theta(b))$ where $\sigma, \tau$ are disjoint $(n-1)$-simplices in $M_{n-1}$, and $a, b$ are distinct points of $M_{0}$. On the other hand $U\left(M_{n-1}\right)$ consists of all (2n-1)-simplices $\sigma \circ \theta(\tau), \sigma \cap \tau=0, U\left(M_{0}\right)$, of all 1-simplices $a \circ \theta(b), a \neq b$. Therefore $V\left(U\left(M_{n-1}\right), U\left(M_{0}\right)\right)$ consists of all $(2 n+1)$ simplices of the form $(\sigma \circ \theta(\tau)) \circ(a \circ \theta(b))$. From the simplicial definition of join there is an obvious homeomorphism of $U\left(V\left(M_{n-1}, M_{0}\right)\right)$ onto $V\left(U\left(M_{n-1}\right), U\left(M_{0}\right)\right)$ given by a vertex isomorphism. The antipodal map $\theta$ is well defined in both spaces and antipodal pairs in one correspond to antipodal pairs in the other by the vertex isomorphism.

Under the induction hypothesis and the case $n=0$, we have homeomorphisms $h_{n-1}, h_{0}$ of $S^{2 n-1}, S^{1}$ onto $U\left(M_{n-1}\right), U\left(M_{0}\right)$, respectively. We consider $S^{2 n-1}$ and $S^{1}$ imbedded in $E^{2 n+2}$ exactly as in the proof of Lemma 1. Using the homeomorphism $\phi$ described there, we have $\phi: V\left(S^{2 n-1}, S^{1}\right) \rightarrow S^{2 n+1} \subseteq E^{2 n+2}$. Now we define $h_{n}^{\prime}: V\left(S^{2 n-1}, S^{1}\right)$ $\rightarrow V\left(U\left(M_{n-1}\right), \quad U\left(M_{0}\right)\right)$ by $h_{n}^{\prime}((x, y, t))=\left(h_{n-1}(x), \quad h_{0}(y), t\right)$ $=(1-t) h_{n-1}(x)+t h_{0}(y)$, and the image point may be considered to be in $U\left(M_{n}\right)$ by the identification mentioned above.

Let $z$ and $-z$ be antipodal points of $S^{2 n+1}$. There is a point $(x, y, t)$ in $V\left(S^{2 n-1}, S^{1}\right)$ so that $\phi((x, y, t))=(1-t)^{1 / 2} x+t^{1 / 2} y=z$. It follows that 
$-z=(1-t)^{1 / 2}(-x)+t^{1 / 2}(-y)=\phi((-x, \quad-y, t))$. By hypothesis $\theta\left(h_{n-1}(x)\right)=h_{n-1}(-x) ;$ since in addition $\theta\left(h_{0}(y)\right)=h_{0}(-y)$,

$$
\begin{aligned}
\theta\left(h_{n}^{\prime} \phi^{-1}(z)\right) & =\theta\left((1-t) h_{n-1}(x)+t h_{0}(y)\right)=(1-t) h_{n-1}(-x)+t h_{0}(-y) \\
& =h_{n}^{\prime} \phi^{-1}(-z) .
\end{aligned}
$$

The argument may clearly be reversed, so we may set $h_{n}=h_{n}^{\prime} \phi^{-1}$.

THEOREM. Suppose $C$ is an n-dimensional complex so that there is a homeomorphism of $S^{2 n+1}$ onto $L(C)$ under which antipodal points of $S^{2 n+1}$ are carried onto symmetric points in $L(C)$. If $f$ is a mapping of $|C|$ onto a metric space $D$ so that for any pair $\sigma, \tau$ of disjoint $n$ simplices in $C, f(\sigma) \cap f(\tau)=0$, then $D$ is absolutely knotted with respect to $E^{2 n+1}$.

Proof. $L(C) \subseteq V(|C|, p) \times V(|C|, p)$. $f$ induces a mapping $F$ of $V(|C|, p) \times V(|C|, \quad p)$ onto $V(D, p) \times V(D, p)$ defined by $F((x, p, t),(y, p, s))=((f(x), p, t),(f(y), p, s))$. Let us denote by $\mathscr{L}$ the image of $L(C)$ under $F$.

Suppose $\alpha \in \sigma \in C$ and $\beta=(x, p, t), x \in \tau \in C$, and $\sigma, \tau$ are disjoint $n$-simplices. Then $z=F(\alpha, \beta)$ and $z^{\prime}=F(\beta, \alpha)$ are clearly symmetric in $\mathcal{L}$. If we assume that $z=z^{\prime}$, more explicitly we have $(f(\alpha),(f(x), p, t))$ $=((f(x), p, t), f(\alpha))$. This means $f(\alpha)=(f(x), p, t)$ which in turn implies $t=0$ or $f(\alpha)=f(x) \in f(\sigma) \cap f(\tau)$, a contradiction. Thus symmetric points in $L(C)$ are carried onto distinct symmetric points in $\mathscr{L}$.

Now let $\phi$ be a mapping of $V(D, p)$ into $E^{2 n+1}$. There is a map $\psi$ of $\mathscr{L}$ into $E^{2 n+1}$ which takes a point $(\gamma, \delta)$ of $\mathscr{L}$ into the endpoint of $W$, the vector from $\phi(\gamma)$ to $\phi(\delta)$, translated to the origin so that its starting point is at the origin. $\psi(\gamma, \delta)$ and $\psi(\delta, \gamma)$ are symmetric with respect to the origin of $E^{2 n+1}$.

We denote by $h$ the homeomorphism of $S^{2 n+1}$ onto $L(C)$ assumed in the hypotheses. If $x,-x$ are antipodal in $S^{2 n+1}, F h(x)$ and $F h(-x)$ are distinct symmetric points in \&. By the Borsuk-Ulam Theorem there exists an antipodal pair $x,-x$ in $S^{2 n+1}$ so that $\psi F h(x)=\psi F h(-x)$; since the image points are also, as we have observed previously, symmetric about the origin, it follows that $\psi F h$ maps $x$ and $-x$ into the origin.

Let $F h(x)=(\gamma, \delta)$ so $F h(-x)=(\delta, \gamma)$. Since $\psi(\gamma, \delta)$ is the origin, $\phi(\gamma)=\phi(\delta)$. If $\gamma \in D, \delta \in D$ implies $\phi$ is not one-to-one on $D$; if $\delta$ is a point of $V(D, p)-D, \phi(D) \cap \phi(V(D, p)-D) \neq 0$. Accordingly $D$ is absoutely knotted with respect to $E^{2 n+1}$.

5. Remarks and questions. Some questions may be raised in view of the preceding results. 
Question 1. Is there a decomposition of $E^{3}$ into $n$ circles, $n<6$, and points not on the circles so that the decomposition space cannot be imbedded in $E^{4}$ ? The author conjectures that if $n=4$ the answer is no. The results of Bing-Curtis and Goblirsch definitely settle the question in the negative for $n<4$. An example of a collection of circles for which the question is open is one of five circles in $E^{3}$ so that each circle links exactly two others.

QUESTION 2. If each nondegenerate element is a polyhedral simple closed curve rather than a circle, what is the least number of nondegenerate elements so that the decomposition space cannot be imbedded in $E^{4}$ ? It would be useful here to know broad conditions under which a collection of tame simple closed curves is equivalent in $E^{3}$ (under a global homeomorphism) to a collection of circles.

QUestion 3. In Question 2 allow one or more of the curves to be wildly imbedded in $E^{3}$.

\section{REFERENCES}

1. R. H. Bing and M. L. Curtis, Imbedding decompositions of 3-space in 4-space, Proc. Amer. Math. Soc. vol. 11 (1960) pp. 149-155.

2. R. Flores, Ueber n-dimensionale Komplexe, die im $R_{2 n+1}$ absolut selbstverschlungen sind, Ergebnisse eines Mathematischen Kolloquiums, vol. 6 (1933-34) pp. 4-6.

3. R. P. Goblirsch, On decompositions of 3-space by linkages, Proc. Amer. Math. Soc. vol. 10 (1959) pp. 728-730.

4. R. H. Rosen, Imbeddings of decompositions of 3-space, doctoral dissertation, University of Wisconsin, August, 1959, pp. 1-38.

UNIVERSITY OF MICHIGAN AND UNIVERSITY OF WISCONSIN 